\title{
Design and Research of Electric Automation Control System based on Chaotic Algorithm
}

\author{
Guangmei Hai ${ }^{a}{ }^{*}$ and Tao Jin ${ }^{\mathrm{b}}$ \\ ${ }^{a}$ Electrical and Automation Engineering Department, Jiangsu Maritime Institute, Nanjing, 210000, China \\ ${ }^{b}$ Production Safety Department, Nanjing Weifu Jingning Limited Company, Nanjing, 210000, China
}

\begin{abstract}
The development of computer technology has led to many intelligent algorithms; the chaos algorithm is one of them. It has played a great role in promoting many fields with its superior performance. The electric power control system of electrical automation equipment can not only optimize the control system but also minimize the consumption of electrical energy. PID controller is often used in the design of the power control system. The control effect of PID controller is greatly influenced by the parameters. The traditional parameter setting method is more complicated and the setting is more difficult. Therefore, a method of optimal design of the power control system for electrical automation equipment based on chaos theory is proposed.
\end{abstract}

Keywords: chaos algorithm; electrical automation; power control; intelligent algorithm

(Submitted on November 5, 2018; Revised on December 3, 2018; Accepted on January 6, 2019)

(C) 2019 Totem Publisher, Inc. All rights reserved.

\section{Introduction}

In recent years, the development and breakthrough of computer network technology and electrical automation technology have made the application of electrical automation equipment more and more widespread and extensive. The design of electrical automation control systems for electrical automation equipment has also been perfected [1]. For the design of the power control system, each link must be strictly controlled to avoid internal differences, and to ensure that the overall performance of the control system is fully utilized. The scientific power control system design can accurately measure the power of the power system to achieve the safe use of electrical energy transmission and the effective control of energy consumption [2]. The control system optimization technology is to solve and optimize various problems in the project. The solution and optimization of these problems can be abstracted as a mathematical model. It is common that this kind of optimization design technology is applied in designing control systems and setting equipment parameters [3]. The PID controller is a classic controller. When the PID controller is used in the power control system, the control effect is closely related to the parameter setting. Therefore, for the PID controller, it can be understood as parameter-controlled technology [4]. In the power control system, the realization of the PID controller's automatic control is based on setting the controller parameters [5]. However, the traditional parameter setting process for PID is extremely cumbersome. It needs to consider the object's requirements for parameters and perform transition calculations and tests [6]. The completion of this process requires professional and experienced technicians to match the controller with different types of power control systems. This virtually increases the difficulty of parameter adjustment and setting; therefore, it is necessary to optimize the PID controller parameter adjustment and setting process [7].

\section{Start of the Art}

The chaos search method is an improved control system optimization algorithm. The so-called chaos theory is essentially a study of nonlinear problems. It is about the unification of order and disorder, determination, and uncertainty. In recent years, chaos theory has been widely used in various fields [8]. In the solution and discussion of practical problems, the application

\footnotetext{
* Corresponding author.

E-mail address: hgm_jshs@126.com
} 
of electric power control systems in electrical automation equipment has also been continuously applied [9]. The theoretical basis of chaos theory had been introduced, and its application in the design of power automation system for electrical automation equipment had been discussed. [10]. Chaos is a universal, non-linear phenomenon, similar to randomness, but there is a certain internal law that can summarize the characteristics of chaos, namely, randomness, ergodicity, and regularity [11]. Randomness means that under certain conditions, the result is not affected by the appearance factor. Ergodicity means that during the process of random simulation, some states in the range are not repeated, the law of regularity means that although chaos has similar random characteristics, it has certain and intrinsic laws. In the control system optimization, chaos theory is mainly used to search through chaos search and incomplete search chaos search. The former is based on ergodicity and can avoid the occurrence of local minima [12]. This requirement for the optimization problem is highly adaptable [13]. It only requires the establishment of functions without the need for continuous or differentiability. Therefore, the former is difficult to express with mathematical models. It is more applicable. The latter directly uses chaotic variables as the variables used and searches directly. The search for chaotic variables is based on the chaos's own laws and characteristics, which increases the probability of obtaining the optimal solution [14].

\section{Methodology}

\subsection{Chaos Optimization Model}

Chaos refers to a phenomenon that exhibits randomness at a certain level. It is mainly used in certain deterministic systems. These systems have very sensitive dependence on the initial values generated, and the application fields of chaos are extremely wide; almost all of the natural sciences are involved. This is because, for many scientific fields, there is a certain degree of chaos. When an event occurs, it has a certain degree of randomness. Therefore, it can also be viewed as a chaotic phenomenon [15]. Although the chaotic system will exhibit an unordered state in its macroscopic performance, when statistical analysis of these states is performed using statistics, it is found that the chaotic system also has certain rules. In the process of using the chaotic system to analyze the randomness of the dynamic system, it is obvious that the chaos has a certain regularity and ergodicity under the set conditions [16]. The chaotic system has its inherent randomness. This randomness is similar to a stochastic system and shows a chaotic appearance without human intervention. The regularity states that chaos is constrained; it is random within a certain range but not completely random. Therefore, even if it is a chaotic system, it is subject to certain certainty factors. For ergodicity, it means that when the chaotic system is in a random state, the states can only be in different states, and the same state will not appear many times. The traversal feature is a very important optimization mechanism in the chaotic system [17]. When the algorithm is used to optimize, it can avoid the optimization result falling into a local optimum. Therefore, the chaos algorithm is an excellent optimization algorithm, which has played a significant role in the development of many fields. The conceptual diagram of the chaos algorithm is shown in Figure 1.

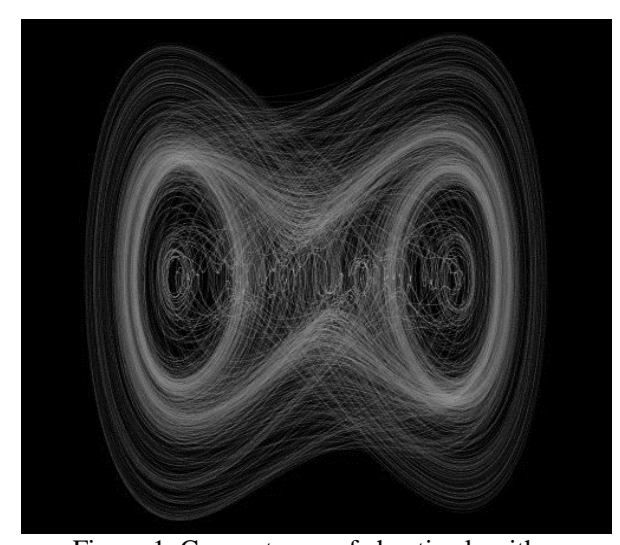

Figure 1. Concept map of chaotic algorithm

The ergodic definition formula is shown in Equation (1):

$$
\lim _{N \rightarrow \infty} \frac{1}{N} \sum_{n=1}^{N} G\left(x_{n}\right)=\int \rho(x) G(x) \mathrm{d} x
$$

Where $\rho(x)$ represents the density distribution function of the map: 
Logistic mapping formula is shown in Formula (2):

$$
y(k+1)=\mu y(k)(1-y(k))
$$

The calculation formula of Logistic map transformation is shown in Formula (3):

$$
y(k)=\sin ^{2}(\pi t(k) / 2)
$$

The flow chart of the chaos optimization algorithm is shown in Figure 2:

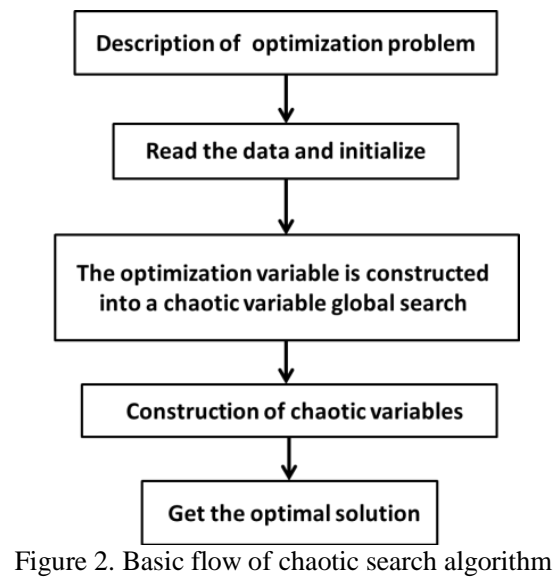

This section mainly introduces the description of the problem, variable transformation, global rough search, and partial fine search. The chaotic sequence has no repeatability. According to this rule, the chaotic sequence traverses all states within a certain range. This is the so-called orbital ergodicity and is based on the optimization of chaotic functions. When selecting chaotic variables, it is extremely important to closely analyze the optimization of chaotic variables. Combining the selected chaotic variable with the variable to be optimized, mapping and describing the range of ergodicity of chaotic motion, the mapping will be placed in the value interval of the variable to be optimized, using ergodicity and ergodic search to optimize search chaos variable. This search method is significantly better than the random search method. There are two main stages in the search process [18]. The first is the generation of iterative maps. The chaotic variables have ergodicity. Therefore, chaotic variables contain ergodicity orbits. This characteristic is used to analyze the problems and search for chaotic variables. This phase is the rough search phase [19]. When the termination condition is satisfied, the state obtained through the search is closed. Moreover, the optimal solution of the problem can be achieved as long as the search time is sufficient. At the same time, this stage can completely serve as the starting point for the next stage. The second stage takes the first stage as the starting point, adding certain disturbances to this stage and searching for local details. The sign of completion in this stage is the completion or satisfaction of the algorithm or criterion. The additional disturbance includes the chaotic variable itself, based on Cauchy distribution, Gaussian distribution, uniformly distributed random variables, and algorithms based on gradient descent for relevant variables of the offset.

\subsection{Chaotic Optimization Algorithm based on Power Function Carrier}

At present, the basic idea of the chaos optimization algorithm is to use a chaotic search algorithm based on the secondary chaotic carrier proposed by Li Bing et al. It mainly uses the ergodicity of chaotic motion. The chaos optimization algorithms in the future are based on this method [20]. This algorithm is based on the idea of two-stage optimization. The carrier-like method is used to introduce the chaotic variables generated by Logistic maps into the optimal variables. At the same time, the traversal range of chaotic motions is transformed into the domain of optimization variables. Then, chaotic variables are used for searching.

The logistic mapping formula is shown in Equation (4):

$$
x_{n+1}=\mu x_{n}\left(1-x_{n}\right)
$$


After a certain number of iterations, a large number of orbital points (chaotic sequences) are distributed on the $(0,1)$ line segment and the orbital probability density function according to the orbital probability density function is shown in Equation (5):

$$
\rho(x)=\frac{1}{\pi \sqrt{x(1-x)}}
$$

The orbital point interval statistics method is used to calculate the actual orbital density distribution function. The specific method is as follows: The specific form of the mapping is known, and the $N$ orbit points are obtained from the iteration of the mapping function from the arbitrary initial point. Dividing the mapping domain $\left[x_{l}, x_{h}\right]$ into $\mathrm{n}$ inter cells, statistics on the number of track points in the $\mathrm{n}$ inter cell area are $N_{i}(i=1,2, \cdots, n)$ respectively. By normalizing the number of track points obtained by statistics, we get the probability density of statistical orbits between the $i$ cells as shown in Formula (6):

$$
\rho(i)=\frac{N_{i} / N}{\left(x_{h}-x_{l}\right) / n}
$$

The orbital probability density distributions according to Equations (5) and (6) are basically the same, so the statistical method can be used to qualitatively describe the probability density distribution of chaotic orbits.

To examine the ergodicity of the Logistic map, iterate 3000 times with $x_{10}=0.43255$ and $x_{20}=0.91623$ as initial values. The small circle in the figure represents a point in the two-dimensional space, as shown in Figure 3:

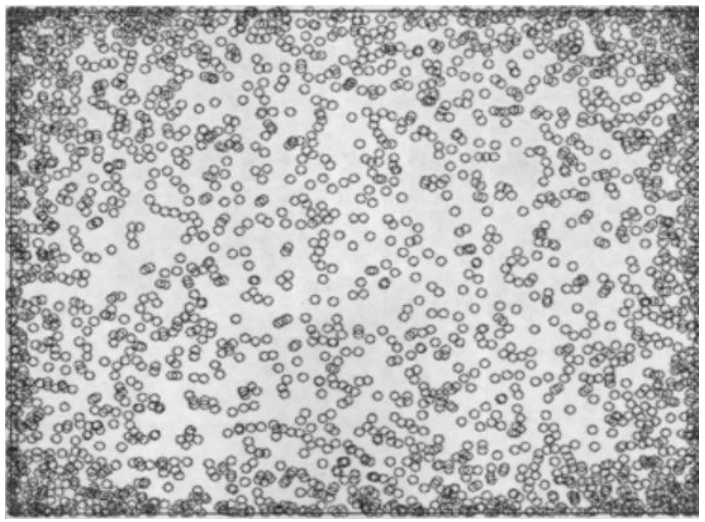

Figure 3. Ergodicity of Logistic mappings

The probability distribution density function of Logistic map is shown in Figure 4.

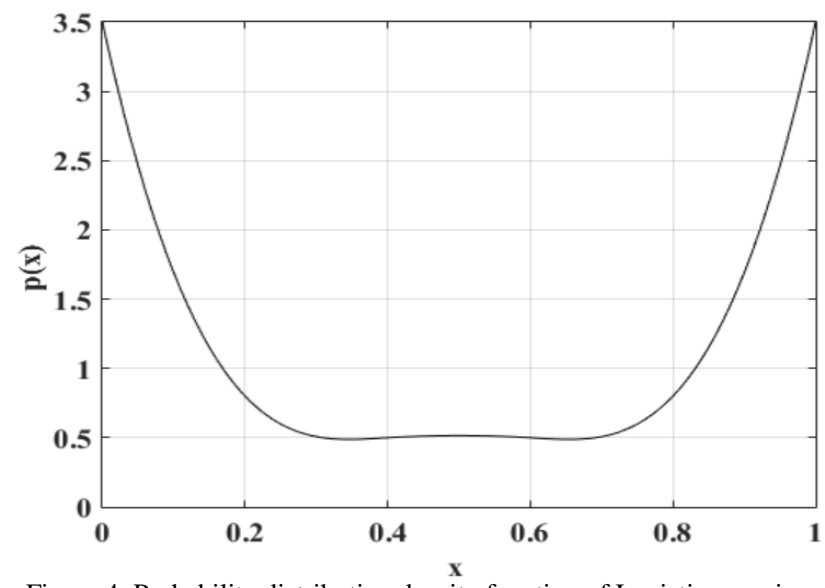

Figure 4. Probability distribution density function of Logistic mappings 
It can be seen from Figure 2 that although the chaotic variables generated by Logistic maps are ergodicity, due to the uneven distribution of orbital points (the ends of the interval are much denser than the interior points of the interval), the ergodicity is affected. As can be seen from Figure 3, the distribution characteristics of the Logistic map chaotic sequence are more uniform in the middle, more than two in number, and the number of endpoints is infinite. Therefore, with chaos optimization, many searches are performed at both ends of the design variable space. When the global best point is not at both ends of the design variable space, it will not be conducive to finding the global optimum.

\subsection{Chaos Optimization Algorithm for Single Variable System Optimization Control}

In view of the good performance of the chaos optimization algorithm, it was applied to the optimal control of the univariate turbine regulating system and the turbine regulating system. The single-neuron adaptive PID controller parameters of the hydro turbine regulation system are optimized by the chaotic optimization method of the secondary carrier. The hybrid chaos optimization method combined with the accelerated chaotic search and variable-scale strategy is used to optimize the parameters of the fuzzy immune PID controller for the steam turbine governing system. The simulation results show that this method effectively achieves the optimal tuning of the controller parameters and significantly improves the control quality of the turbine regulating system and the turbine regulating system. Commonly used turbines are shown in Figure 5:

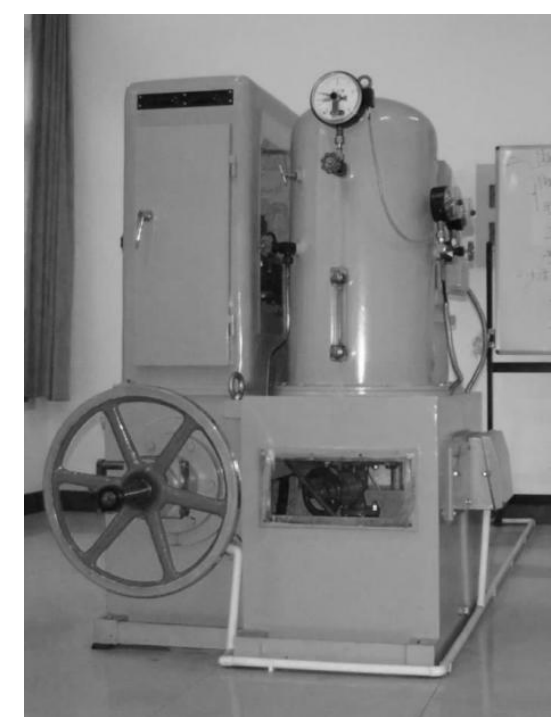

Figure 5. Commonly used hydraulic turbines

The turbine regulation system is a feedback control system consisting of pressure diversion, a hydraulic turbine, a generator and a governor. If an analog governor is used, the turbine adjustment system is a continuous system. The turbine regulation system is a certain value control system under no-load and stand-alone load operating conditions. When the capacity of the power grid is large and the change of output power of a unit operating in parallel cannot affect the frequency of the power system, the feedback of the speed of the turbine regulating system is almost ineffective, which is equivalent to the open-loop state. In this case, the governor completes the function of the servo system. The single neuron self-adaptive intelligent PID controller composed of single neurons with self-learning and self-adaptive capabilities not only has a simple structure, but also can adapt to environmental changes and has important features such as less on-site adjustment parameters and easier on-site debugging. The robustness can greatly improve the dynamic quality of a typical nonlinear time-varying object, ensuring that the control system operates in an optimal state and the control quality is obviously better than the conventional PID controller.

The controlled object of the turbine regulation system is a system that is highly nonlinear, highly complex, highly uncertain, and contains non-minimum phase links. At present, the most widely used turbine is still a typical PID control, mainly because this control strategy is robust and straightforward. To some extent, it is more difficult to completely change this control method and establish and apply a completely new control strategy. Therefore, it is particularly important to study how to improve the existing control methods and have practical value. The use of intelligent control to make the turbine governor intelligent is one of the effective methods to achieve a good control function of the turbine regulating system. Judging from the characteristics of hydropower units, the use of fuzzy control is considered to be an effective solution. Aiming at the problem that the conventional PID controller cannot perform online self-tuning, a fuzzy self-tuning of PID parameters is proposed. By combining the fuzzy control and neural network and aiming at the characteristics of non- 
linearity and large range of structural parameters of the hydraulic turbine regulating system, a new type of turbine control strategy-FNNS (Fuzzy Neural Network System) and variable parameter control system were proposed.

\section{Result Analysis and Discussion}

Turbine speed control system control actuators have a dead zone of 0.25 and a saturation zone of 0.75 with a total process lag of $6 \mathrm{~s}$. The sampling period takes $T=1 \mathrm{~s}$, the controlled object is discretized, and a single neuron adaptive PID controller based on chaos optimization is used for control. The traditional PID controller takes values of $k p=0.7, k i=1$, and $k d=$ 0.04. The parameters of the single neuron adaptive PID controller are set to $w(0)=0.34, w 2(0)=0.32, w 3(0)=0.33$, and different values are used for the integral $\mathrm{I}$, the ratio $P$, and the differential $D$, respectively. Learn the rate so that different weights can be adjusted separately. The choice of the $K$ value is very important. The bigger $K$ is, the better the rapidity is. If the overshoot is too large, it may make the system unstable. When the delay of the controlled object is large, $K$ must be reduced to ensure the stability of the system. If the value of $K$ is too small, the rapidity of the system will be degraded. According to experience, $K=0.02, P=2$, and $Q=1$ are selected. The simulation results are shown in Figure 6:

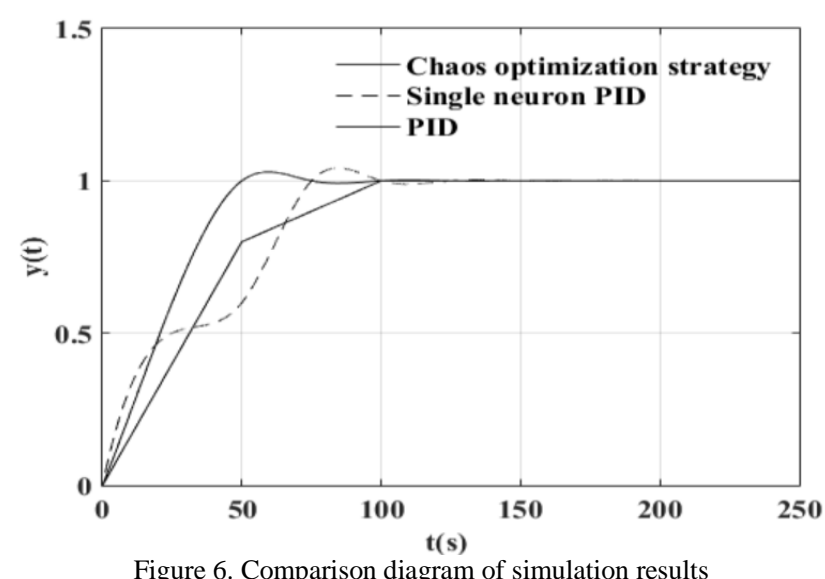

It can be seen from Figure 6 that when using a single neuron PID control method, the system has a long adjustment time despite the absence of overshoot. The traditional PID control not only responds slowly but also oscillates and sometimes overshoots and the system has a long adjustment time despite the absence of overshoot. When using the chaos optimization method to optimize the controller parameters, the control system is stable. There is basically no oscillation and no overshoot and has a fast response and short adjustment time. Thus, for a hydro-turbine speed regulation system with a nonlinear link, the single neuron adaptive PID controller based on chaos optimization is significantly better than the single neuron adaptive PID controller and the traditional PID controller. In view of the complex characteristics of turbine regulating objects, a single neuron adaptive PID control system based on chaos optimization strategy was proposed. Simulation results show that compared with the traditional PID control and single neuron PID control, this control strategy has the advantages of stability, small overshoot, fast response, good adaptability, simple structure and easy implementation, etc., in order to solve the PID controller. The global optimal design of the parameters provides an effective method.

The chaos algorithm makes full use of the characteristics of accelerated chaotic search and variable-scale methods to avoid the time wasted in calculating unnecessary bad points in chaos optimization, making the initial point of the variablescale method to be in a more favorable position and for it to jump out of the variable-scale method to be in a more favorable position. The optimal conditions were created and finally, a satisfactory optimal solution was obtained. Simulation results show that compared with the traditional PID control and fuzzy immune PID control, this control strategy has the advantages of stability, small overshoot, fast response, short adjustment time, good adaptability, simple structure, easy implementation, and high optimization efficiency.

\section{Conclusion}

The chaotic algorithm has a good application prospect in many fields. The chaotic algorithm was applied to the design research of electrical automation equipment electrical control system, which can play a great role in promoting the electrical control systems of electrical automation equipment. The algorithm is an excellent optimization algorithm so that it can solve some optimization problems in the store control system. The chaos optimization model, which is an excellent mathematical model, was first established. In addition, this kind of model can be used to conduct more in-depth research on the electrical 
control system. A chaos optimization algorithm based on power function carrier was also proposed. This algorithm can effectively solve the optimization problem of some parameters in electrical control system. The extensive application of electrical automation equipment has greatly improved the production efficiency of the electrical industry. The design of the electrical automation system power control system is of the highest priority. The optimal design method of power control systems based on the chaos theory can reduce the calculation amount and has strong operability, which simplifies the parameter setting process of PID controller. The scientific nature of this design method will surely make it widely used in the design of electrical automation equipment power control systems. With the wide application of this design method, its theoretical perfection and scientific will also be improved and more in-depth research will be conducted.

\section{References}

1. C. Z. Shi, Z. Dou, Y. Lin, and W. W. Li, "Dynamic Threshold-Setting for RF-Powered Cognitive Radio Networks in NonGaussian Noise," Physical Communication, Vol. 27, pp. 99-4105, April 2018

2. M. L. C. Peixoto, E. G. Nepomuceno, S. A. M. Martins, and M. J. Lacerda, "Computation of the Largest Positive Lyapunov Exponent using Rounding Mode and Recursive Least Square Algorithm," Chaos, Solitons \& Fractals, Vol. 112, pp. 36-43, July 2018

3. B. S. Rees and K. B. Gallagher, "Overlapping Community Detection using a Community Optimized Graph Swarm," Social Network Analysis and Mining, Vol. 2, No. 4, pp. 405-417, December 2012

4. Y. Shen, "A Chaos Genetic Algorithm based Extended Kalman Filter for the Available Capacity Evaluation of Lithium-Ion Batteries," Electrochimica Acta, Vol. 264, pp. 400-409, February 2018

5. Ünal Çavuşoğlu, S. Kaçar, A. Zengin, and I. Pehlivan, "A Novel Hybrid Encryption Algorithm based on Chaos and S-AES Algorithm," Nonlinear Dynamics, Vol. 92, No. 4, pp. 1745-1759, June 2018

6. Y. Shen, "Improved Chaos Genetic Algorithm based State of Charge Determination for Lithium Batteries in Electric Vehicles," Energy, Vol. 152, pp. 576-585, June 2018

7. T. Manos and R. E. G. Machado, "Chaos and Dynamical Trends in Barred Galaxies: Bridging the Gap Between N-Body Simulations and Time-Dependent Analytical Models," Monthly Notices of the Royal Astronomical Society, Vol. 438, No. 3, pp. 2201-2217, March 2014

8. M. E. J. Newman, "Fast Algorithm for Detecting Community Structure in Networks," Physical Review E, Vol. 69, No. 6, pp. 066133, June 2004

9. A. S. Mohammed, A. E. Amrahov, and F. V. Elebi, "Bidirectional Conditional Insertion Sort algorithm: An Efficient Progress on the Classical Insertion Sort," Future Generation Computer Systems, Vol. 71, pp. 102-112, June 2017

10. Z. H. Deng, J. W. Xu, X. Q. Li, and F. Huang, "Robust Adaptive Beam-Forming Optimization Method based on DiagonalLoading and MSE Criterion," Chaos, Solitons \& Fractals, Vol. 108, pp. 87-93, March 2018

11. L. X. Li, H. Zhang, H. P. Peng, and Y. X. Yang, "Nearest Neighbors-based Density Peaks Approach to Intrusion Detection," Chaos, Solitons \& Fractals, Vol. 110, pp. 33-40, May 2018

12. E. Wang, C. Y. Jia, G. Tong, P. P. Qu, X. Y. Lan, and T. Pang, "Fault Detection and Isolation in GPS Receiver Autonomous Integrity Monitoring based on Chaos Particle Swarm Optimization-Particle Filter Algorithm," Advances in Space Research, Vol. 5, No. 1, pp. 1260-1272, March 2018

13. J. G. Sun, W. S. Wang, L. Kou, Y. Lin, L. G. Zhang, Q. G. Da, et al., "A Data Authentication Scheme for UAV Ad Hoc Network Communication,” Journal of Supercomputing, No. 8, pp. 1-16, 2017

14. C. A. III Pope, R. T. Burnett, M. J. Thun, E. E. Calle, D. Krewski, K. Ito, et al., "Lung Cancer, Cardiopulmonary Mortality, and Long-Term Exposure to Fine Particulate Air Pollution," Jama, Vol. 287, No. 9, pp. 1132-1141, March 2002

15. X. H. Wang, X. H. Bi, G. Y. Sheng, and J. M. Fu, "Chemical Composition and Sources of PM10 and PM2.5 Aerosols in Guangzhou, China," Environmental Monitoring and Assessment, Vol. 119, No. 1-3, pp. 425-439, June 2006

16. Y. Zhang, "The Unified Image Encryption Algorithm based on Chaos and Cubic S-Box," Information Sciences, Vol. 450, pp. 361-377, June 2018

17. F. Z. Zhao, G. C. Xiao, M. Liu, and D. S. Yang, "A Fast Sorting Strategy based on a Two-Way Merge Sort for Balancing the Capacitor Voltages in Modular Multilevel Converters," Journal of Power Electronics, Vol. 17, No. 2, pp. 346-357, November 2017

18. J. Wang, Y. Y. Luo, L. Y. Tang, and P. Ge, "Modeling a Combined Forecast Algorithm based on Sequence Patterns and Near Characteristics: An Application for Tourism Demand Forecasting," Chaos, Solitons \& Fractals, Vol. 108, pp. 136-147, March 2018

19. Z. Ye, S. Hu, and J. Yu, "Adaptive Clustering Algorithm for Community Detection in Complex Networks," Physical Review E, Vol. 78, No. 4, pp. 046115, October 2008

20. J. Z. Xu, Y. L. Xu, Y. C. Zhao, C. Y. Zhao, H. Ding, and Y. Zhang, "Linear Time Complexity Sorting Algorithms for Electromagnetic Transient Simulation of MMC-HVdc System," Iet Generation Transmission \& Distribution, Vol. 11, No. 16, pp. 4059-4067, 2017 